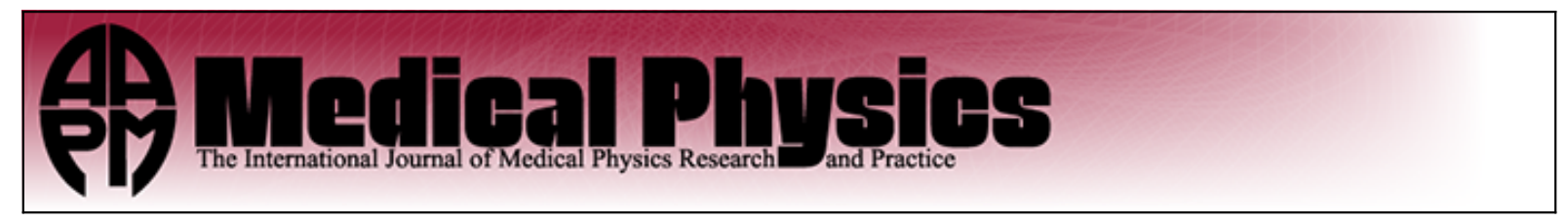

\title{
Application of spherical diodes for megavoltage photon beams dosimetry
}

Benigno Barbés, Juan D. Azcona, Javier Burguete, and Josep M. Martí-Climent

Citation: Medical Physics 41, 012102 (2014); doi: 10.1118/1.4837178

View online: http://dx.doi.org/10.1118/1.4837178

View Table of Contents: http://scitation.aip.org/content/aapm/journal/medphys/41/1?ver=pdfcov

Published by the American Association of Physicists in Medicine

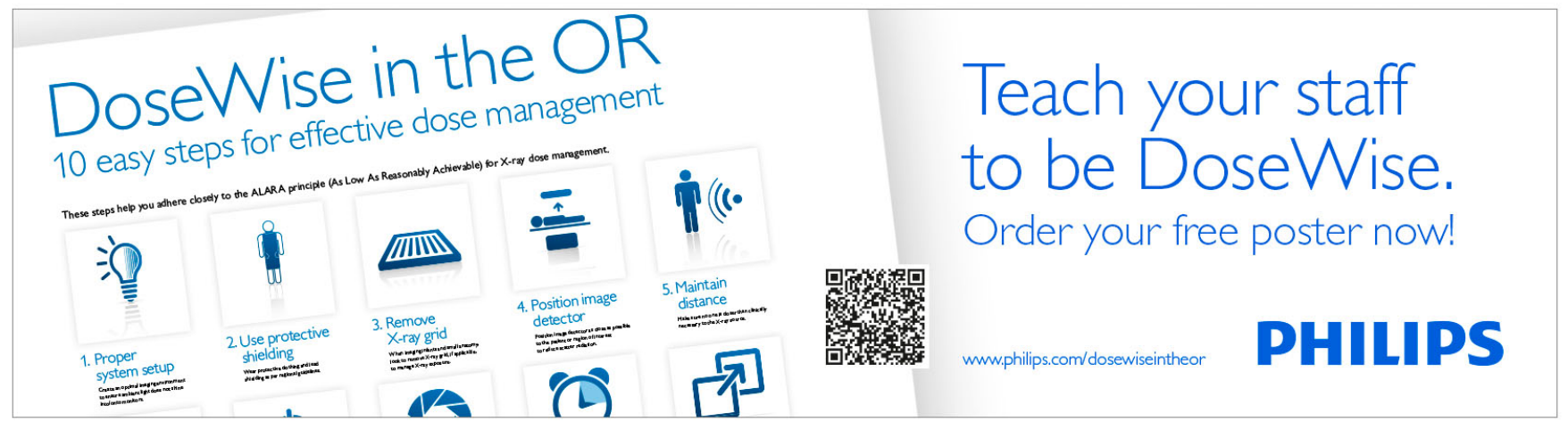




\title{
Application of spherical diodes for megavoltage photon beams dosimetry
}

\author{
Benigno Barbés ${ }^{a}$ \\ Servicio de Oncología Radioterápica, Clínica Universidad de Navarra, Avda. Pío XII, 36, E-31008 Pamplona, \\ Navarra, Spain \\ Juan D. Azcona \\ Department of Radiation Oncology, Stanford University, Stanford, California 94305 and Servicio de Oncología \\ Radioterápica, Clínica Universidad de Navarra, Avda. Pío XII 36, E-31008 Pamplona, Navarra, Spain \\ Javier Burguete \\ Departamento de Física y Matemática Aplicada, Facultad de Ciencias, Universidad de Navarra, Irunlarrea 1, \\ E-31008 Pamplona, Navarra, Spain \\ Josep M. Martí-Climent \\ Servicio de Medicina Nuclear, Clínica Universidad de Navarra, Avda. Pío XII 36, E-31008 Pamplona, \\ Navarra, Spain
}

(Received 18 June 2013; revised 23 October 2013; accepted for publication 15 November 2013; published 9 December 2013)

Purpose: External beam radiation therapy (EBRT) usually uses heterogeneous dose distributions in a given volume. Designing detectors for quality control of these treatments is still a developing subject. The size of the detectors should be small to enhance spatial resolution and ensure low perturbation of the beam. A high uniformity in angular response is also a very important feature in a detector, because it has to measure radiation coming from all the directions of the space. It is also convenient that detectors are inexpensive and robust, especially to perform in vivo measurements. The purpose of this work is to introduce a new detector for measuring megavoltage photon beams and to assess its performance to measure relative dose in EBRT.

Methods: The detector studied in this work was designed as a spherical photodiode $(1.8 \mathrm{~mm}$ in diameter). The change in response of the spherical diodes is measured regarding the angle of incidence, cumulated irradiation, and instantaneous dose rate (or dose per pulse). Additionally, total scatter factors for large and small fields (between $1 \times 1 \mathrm{~cm}^{2}$ and $20 \times 20 \mathrm{~cm}^{2}$ ) are evaluated and compared with the results obtained from some commercially available ionization chambers and planar diodes. Additionally, the over-response to low energy scattered photons in large fields is investigated using a shielding layer.

Results: The spherical diode studied in this work produces a high signal $(150 \mathrm{nC} / \mathrm{Gy}$ for photons of nominal energy of $15 \mathrm{MV}$ and 160 for $6 \mathrm{MV}$, after $12 \mathrm{kGy}$ ) and its angular dependence is lower than that of planar diodes: less than 5\% between maximum and minimum in all directions, and $2 \%$ around one of the axis. It also has a moderated variation with accumulated dose (about $1.5 \% / \mathrm{kGy}$ for $15 \mathrm{MV}$ photons and $0.7 \% / \mathrm{kGy}$ for $6 \mathrm{MV}$, after $12 \mathrm{kGy})$ and a low variation with dose per pulse $( \pm 0.4 \%)$, and its behavior is similar to commercial diodes in total scatter factor measurements.

Conclusions: The measurements of relative dose using the spherical diode described in this work show its feasibility for the dosimetry of megavoltage photon beams. A particularly important feature is its good angular response in the MV range. They would be good candidates for in vivo dosimetry, and quality assurance of VMAT and tomotherapy, and other modalities with beams irradiating from multiple orientations, such as Cyberknife and ViewRay, with minor modifications. (0) 2014 American Association of Physicists in Medicine. [http://dx.doi.org/10.1118/1.4837178]

Key words: dosimetry, diode, spherical-symmetry, radiotherapy

\section{INTRODUCTION}

Sophisticated irradiation techniques, such as intensity modulated radiation therapy (IMRT) or volumetric modulated arc therapy (VMAT), entail several challenges as regards their commissioning and quality assurance dosimetry. These techniques are based on small or modulated fields, composed of several irregularly shaped subfields. The use of these small fields presents special characteristics such as partial occlusion of the primary source and lack of lateral charged particle equilibrium. ${ }^{1}$ Furthermore, the detectors employed for their dosimetry cause a perturbation in the measurement because of their finite volume and different density compared to the absorbing medium. Additionally, in modulated fields the detector could be located at a high dose point where several penumbras - from different segments - match. It is, thus, necessary to use smaller dosimeters in order to diminish its perturbation of the signal and measuring conditions and also to further improve the spatial resolution. 
The International Commission on Radiation Units and Measurements (ICRU) Report No. 83 (Ref. 2) points out that there is no single dosimetry system that conveniently measures all the dosimetric properties necessary for patientspecific QA in IMRT treatments. Besides, the dosimetric uncertainties associated with these new techniques demand an exhaustive quality assurance to ensure that the delivered dose agrees with the planned dose. To accomplish this aim, in vivo and 2D array dosimeters are now widely used for verification of planar or rotational therapy plans, and require robust, small, and economic detectors.

According to the recommendations of the International Atomic Energy Agency (IAEA), ${ }^{3}$ ionization chambers are the detector of choice for the measurement of absolute absorbed dose. However, for more than $50 \mathrm{yr}$, silicon semiconductor diodes have been used as radiation detectors. ${ }^{4-7}$ Their higherthan-air electronic density, along with the low average energy required to form a carrier pair inside them, makes it possible to produce in these diodes radiation current densities about 18000 times those of air. ${ }^{8-10}$ This allows a small volume of silicon diode (approximately $10^{-2}-10^{-1} \mathrm{~mm}^{3}$ ) to produce a current that can easily be measured. This high sensitivity (defined as charge collected per unit of absorbed dose) permits their use as very small volume detectors. Other advantages of these diodes are their cost, robustness, and quick response time (microseconds compared to milliseconds of an ion chamber), real-time readout, and stopping power ratios that are nearly energy independent. ${ }^{1}$ These characteristics make these detectors very useful for some special dosimetric applications, such as in vivo dosimetry, ${ }^{11-13}$ dosimetry of small and modulated fields, composite dose distributions, and quality control relative dosimetry. Other small volume detectors are thermoluminescent dosimeters (TLD), ${ }^{14}$ gel dosimeters, ${ }^{15}$ alanine dosimeters using electron paramagnetic resonance (with the drawback of very low sensitivity ${ }^{16}$ ), film dosimetry, mini-plane parallel chamber, ${ }^{17}$ diamond detectors, ${ }^{18}$ dedicated "ultramicro" cylindrical ionization chamber, ${ }^{19}$ metal oxide silicon field-effect transistors (MOSFET) detectors, ${ }^{20}$ and optically stimulated luminescent dosimeters (OSLD). ${ }^{21}$

The physics of charge generation and collection in semiconductor diodes introduces characteristic features that are important for their accurate clinical use. In diodes, the process that determines how many of the mobile charges generated by radiation are collected is not direct recombination, as in ionization chambers, but indirect recombination: a minority carrier is captured by a recombination-generation (RG) centre and then recombines with a majority carrier. ${ }^{11,22}$ This is the reason for the variation of the diode response with the instantaneous dose rate (or dose per pulse), ${ }^{23,24,11}$ with the accumulated dose,,${ }^{9,11,24}$ and with temperature. ${ }^{7}$

There are also other dependencies resulting from detector design. First, there is directional dependence due to diode shape. ${ }^{25,26}$ Second, the detector has a dependence on irradiation energy ${ }^{27-29}$ owing mainly to the materials that surround the die (this is the typical name for the silicon piece in the diode): electrode attachment, protective housing, and buildup, that can contain combinations of metals such as $\mathrm{Al}, \mathrm{Cu}, \mathrm{Sn}, \mathrm{Au}, \mathrm{Ag}, \mathrm{Pb}, \mathrm{W}, \mathrm{Ta}$, and $\mathrm{Fe}$. For photon beam in vivo dosimetry, vendors provide different detectors dedicated to different energy ranges; for electron beams, a single diode model with minimal buildup generally covers the entire clinical energy range. Another important effect is field-size dependence, ${ }^{10,22,30-33}$ which is heavily influenced by the dimensions of the buildup of the diode. It is important to add that both the die and the materials that surround it alter the dose in their surroundings (dose shadow). ${ }^{10,32,34}$

Low angular dependence of dose sensitivity is a key feature for some diode applications, such as in vivo dose or arctherapy dose measurement, ${ }^{26}$ and a diode constructed with spherical symmetry is a good candidate for these applications. In the present study, spherical diodes of $1.8 \mathrm{~mm}$ in diameter were studied to assess their ability to perform dosimetric measurements in radiotherapy high energy photon beams. These were very low cost diodes, designed for the visible light energy spectrum. They have shown a good response in energies of low dose seed brachytherapy $(20-30 \mathrm{keV}),{ }^{35}$ but they had not yet been used for high energy megavoltage (MV) photon beams employed in radiotherapy, to the best of our knowledge. In this paper, we perform the first study of the spherical diode Sphelar ${ }^{\circledR}$ One X03 as an MV photon dosimeter.

The following characteristics of the Sphelar ${ }^{\circledR}$ diode were tested: (1) sensitivity variation with accumulated dose; (2) instantaneous dose rate (or dose per pulse) response; (3) directional dependence; (4) accuracy and precision compared to other dosimeters for large and small fields; (5) behavior compared with that of two different silicon diodes, to analyze advantages and drawbacks; (6) the effect of shielding with a high atomic number material to empirically correct the over-response that silicon diodes exhibit in large fields with a higher proportion of low energy scattered photons; and (7) use of the detector in water, sealed with epoxy resin that does not modify its characteristics. The change of Si diodes with temperature $\left[0.1-0.5 \% /{ }^{\circ} \mathrm{C}\right.$ (Ref. 36)] is a practically negligible effect during an irradiation session (less than $0.1-0.2^{\circ} \mathrm{C}$ ).

Total scatter factors $S_{c p}$ (dose at a reference depth for a given field size divided by the dose at the same point and depth for the reference field size ${ }^{22}$ ) are sensitive to the aforementioned field size dependence. This effect is highly relevant for small field dosimetry and needs to be corrected, ${ }^{37}$ so we chose total scatter factor as the property to compare spherical diodes with other detectors.

\section{MATERIAL AND METHODS}

\section{A. Equipment}

The spherical diode employed in this study is the Sphelar ${ }^{\circledR}$ One X03, manufactured by Sphelar ${ }^{\circledR}$ Power (Sphelar Power Corporation, Kyoto, Japan), a former division of Kyosemi Corporation. ${ }^{38,39}$ Its structure consists of a p-type spherical silicon crystal covered by an n-type silicon spherical shell (see Fig. 1). The spheres are made by dripping molten silicon, allowing the surface tension of the silicon droplets to mould them into single crystal silicon spheres during free-fall. The width of the depletion layer under no bias is estimated to be around $3 \times 10^{-7} \mathrm{~m}$, and the doping level is around 


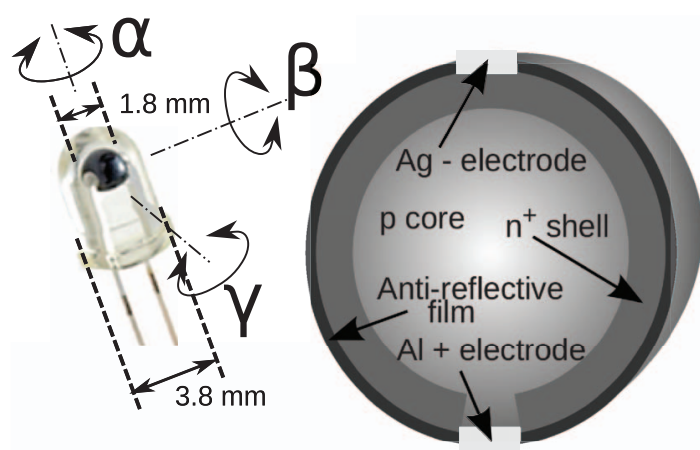

FIG. 1. Dimension and components of the Sphelar ${ }^{\circledR}$ diode.

$10^{22}$ atoms $/ \mathrm{m}^{3}$. Since it can detect radiation coming from all directions in three dimensions, its efficiency is more than three times that of a planar diode of a similar size over isotropic visible light irradiation. ${ }^{40}$ It was designed as a photodiode, but it is also sensitive to ultraviolet (UV) rays and higher energy photons. The diode shows an angular dependence lower than $7 \%$ for solar radiation. ${ }^{39}$ Broisman et al. ${ }^{35}$ found a good response for brachytherapy with I-125 and Pd103 radioactive sources, with energies of 28 and $20 \mathrm{keV}$, respectively, on a PMMA phantom. They performed measurements of dose decay with the distance to the source, reproduction of TG 43 protocol $^{41}$ for polar anisotropy function of a seed, variation of the dose with movements of the seed around the diode (it was lower than 5\%), and accordance with Monte Carlo and TLD measurements. The Sphelar One ${ }^{\circledR}$ is not waterproof, so we covered one of the diodes employed in this work with a thin epoxy resin layer $(0.3-0.4 \mathrm{~mm})$ in the electrode area in order to perform measurements in water. Since the diode's response to visible photons is much higher than that to high energy photons, the diode was covered with a black plastic cap when measuring in water, and the measurements were performed in darkened environment, to prevent visible photons contributing to its signal. Before each measurement, we ensured that the contribution of light contamination to the charge collected by the electrometer (leakage current) was less than $50 \mathrm{pA}$, about $0.1 \%$ of the normal measurement. We used four Sphelar diodes, one of which had resin covering for water measurements.

To test the diode in MV energies such as those used in external radiotherapy with linear accelerators, we employed a Siemens Primus and a Siemens Oncor (Siemens, Munich, Germany), with nominal photon energies of 6 and $15 \mathrm{MV}$ (tissue phantom ratio $\mathrm{TPR}_{20,10}$ of 0.67 and 0.76 , respectively, for both linacs). Multileaf collimators have $1 \mathrm{~cm}$ leaf width at isocenter. The tolerance in the positioning of the leafs is $\pm 1 \mathrm{~mm}$, according to the manufacturer's specifications.

The detectors used for comparisons with the spherical diode were: a Farmer type ionization chamber PTW (PTW-Freiburg, Freiburg, Germany) 30006 with $0.6 \mathrm{~cm}^{3}$ volume; an ionization chamber PTW 31002, with volume of $0.125 \mathrm{~cm}^{3}$; a PTW 31016 chamber (PinPoint 3D) with $0.016 \mathrm{~cm}^{3}$ of volume; a stereotactic diode and an unshielded diode-models SFD and EFD from Scanditronix (Scanditronix Medical AB, Uppsala, Sweden). The active volumes of both diodes are silicon disks of thickness $0.06 \mathrm{~mm}$, the diameter of the disk being $0.6 \mathrm{~mm}$ for the SFD model and $2.0 \mathrm{~mm}$ for the EFD model. The electric charge was collected by a PTW Unidos E electrometer, after a zero drift correction previous to each measurement set with each detector. The external voltage applied to the ionization chambers was $400 \mathrm{~V}$, and no voltage was applied to the diodes.

The measurement media were water purified by inverse osmosis, water equivalent plastic RW3, and acrylic plastic. The water tank was Scanditronix RFA 300; RW3 phantoms were PTW 29672 (composed of square slabs of $30 \times 30 \mathrm{~cm}$ ) and PTW T40015 (head and neck, composed of circular slabs of diameter $20 \mathrm{~cm}$ ); acrylic phantom was a PTW T2966 (20 $\times 20 \times 14.8 \mathrm{~cm})$.

\section{B. Measurement setup}

\section{B.1. Sensitivity}

The measurements of sensitivity variation with accumulated dose were performed in RW3, using photons with nominal energy of 6 and $15 \mathrm{MV}$, a source to surface distance (SSD) of $90 \mathrm{~cm}$, and at the maximum depth $(1.5 \mathrm{~cm}$ for $6 \mathrm{MV}$ and $3 \mathrm{~cm}$ for $15 \mathrm{MV}$ ). The effect of previous irradiation was measured in a spherical diode from 0 to $500 \mathrm{~Gy}$. Another, shorter, series of measures was performed with a diode that had previously received an estimated dose of $190 \mathrm{~Gy}$ with photons with nominal energy of 6 and $15 \mathrm{MV}$. A third diode was irradiated with electrons of 6 and $9 \mathrm{MeV}$, and measurements of sensitivity variation were taken with accumulated doses between 600 and $5400 \mathrm{~Gy}$. The same diode received another $5.4 \mathrm{kGy}$ with a $10 \mathrm{MeV}$ electron beam in an industrial irradiation device afterwards.

\section{B.2. Directional dependence}

There are two setups to measure the directional dependence of a detector: to place it inside a symmetric phantom or to place it on the surface of a flat phantom. Using the second geometry, the angular response of the diode and the scatter and attenuation of the phantom are convolved, so the response of the diode cannot be isolated. ${ }^{26}$ It is useful for evaluating surface measurements, but not for characterization of the diode itself. We used the first setup, placing the Sphelar ${ }^{\circledR}$ diode inside a PTW T40015 cylindrical phantom on its support, aligned with the axis of the gantry. To measure variation with angle $\alpha$ (see Fig. 2 for the $\alpha, \beta$, and $\gamma$ angle convention), the diode and its cable were positioned on the axis of the phantom, using the hole drilled for inserting the PTW 31002 chamber. The difference between $\beta$ and $\gamma$ dependences is due to the asymmetry between anode and cathode (Fig. 1). To measure the dependence with angles $\beta$ and $\gamma$ (Fig. 2), the diode and its cable were placed along the radius of one of the slabs of the phantom. To do this, we used two RW3 semicircular slabs $1 \mathrm{~cm}$ thick and $20 \mathrm{~cm}$ in diameter, in which a hole was mechanized with a numerically controlled tool, to fit in the spherical diode with its coaxial cable between the two pieces (Fig. 2). This formed a circular slab, which replaced one of 

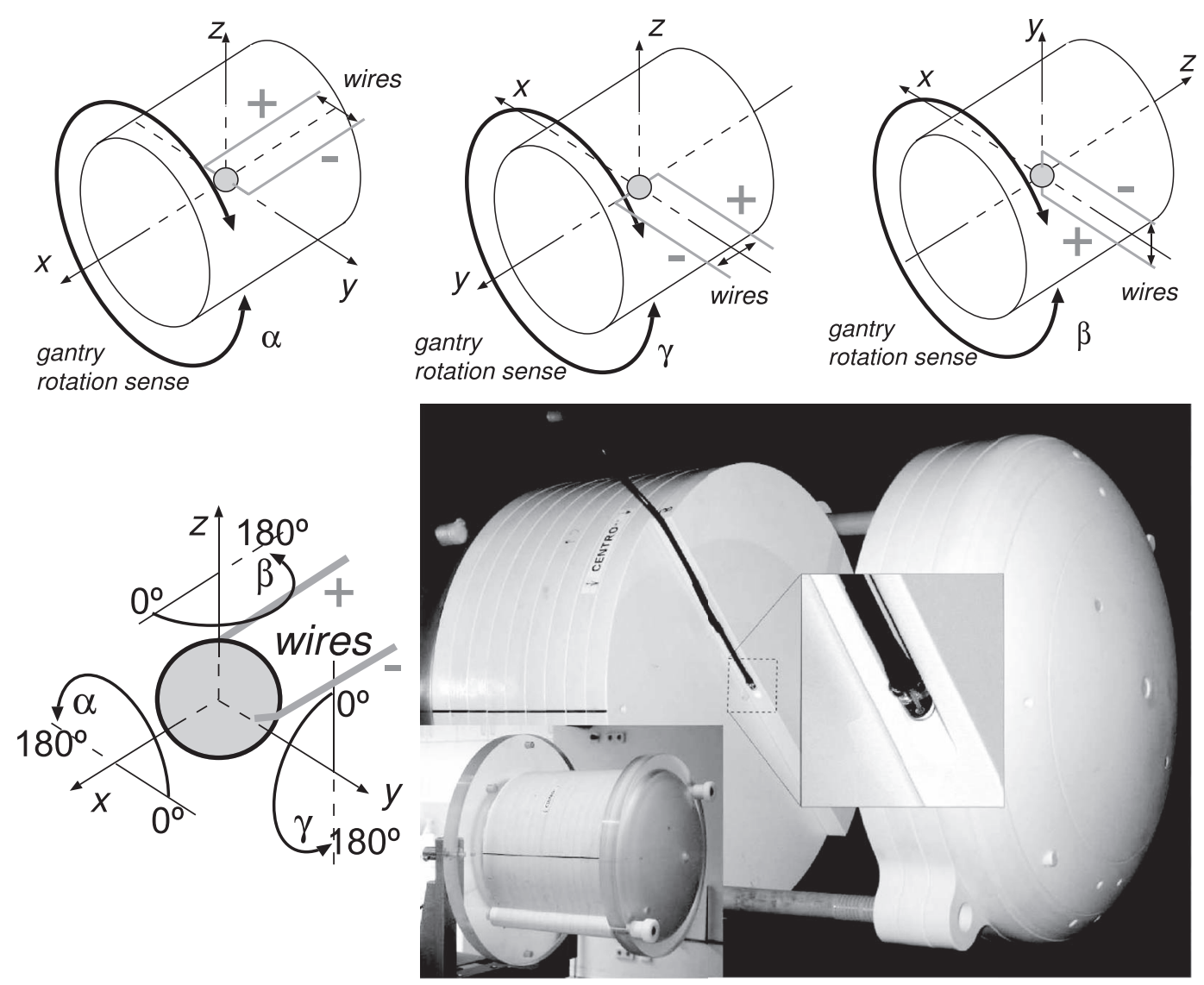

FIG. 2. Diagram of rotations around the diode, and photograph of the phantom designed to measure angular response of the Sphelar around angles $\alpha, \beta$, and $\gamma$.

the slabs of the PTW T40015 cylindrical phantom. The gantry rotation axis was aligned to $Z$ to measure $\beta$ dependence, and was aligned to $Y$ to measure $\gamma$ dependence.

Measurements were taken at surface to axis distance (SAD) of $100 \mathrm{~cm}$, using a $10 \times 10 \mathrm{~cm}$ field, $\mathrm{x}$ ray of nominal energy of $6 \mathrm{MV}$, and $50 \mathrm{MU}$. The spherical diode used for these measurements had previously received $1800 \mathrm{~Gy}$, so sensitivity variation with accumulated dose was very small.

\section{B.3. Dose per pulse}

Measurements of dose per pulse response were made using the acrylic phantom PTW T2966, suitable for tip-to-tip calibrations of therapy chambers. The reference chamber (Farmer PTW 30006) and the spherical diode were irradiated simultaneously, and the dose was obtained via the measurement from the Farmer chamber, applying pressure, temperature, saturation, and beam quality corrections to the chamber measurements. We used a beam with nominal energy of $15 \mathrm{MV}$, keeping a constant dose rate $(500 \mathrm{MU} / \mathrm{s})$ and monitor units (100 MU), placing the chamber and diode at a depth of $5 \mathrm{~cm}$. Instantaneous dose rate was varied by changing the SSD from 70 to $149 \mathrm{~cm}$.

\section{B.4. Total scatter factors}

Measurements of $S_{c p}$ were performed at a SSD of $90 \mathrm{~cm}$, and detector depth of $10 \mathrm{~cm}$, following the recommendations of IAEA ${ }^{3}$. Measurements with the ionization chamber were performed in RW3; measures with SFD and EFD diodes were performed in water; with the spherical diode, we performed a set of measures in water for small fields, and the other measures were performed in RW3.

As discussed below, silicon diodes exhibit an overresponse to $\mathrm{x}$-ray beams in large fields, and have to be compensated. In this paper, we compare measures of Sphelar ${ }^{\circledR} S_{c p}$ with others using an uncompensated commercial diode (the Scanditronix EFD), although neither of them is suitable for such measurements, to study if their responses are similar.

For the planar diodes, the displacements of the effective point of measurement were considered. For the spherical diode, the point of measurement was taken to be the geometric centre of the spherical die. The maximum error in the positioning with this procedure was estimated to be $\pm 0.5 \mathrm{~mm}$ in each direction. The positioning of the ionization chambers in plastic phantom was performed by employing the inserts specifically designated for that purpose for each one of them. The spherical diode was positioned using the insert designed for the PTW 31002 chamber, placing its centre at the centre of the phantom slab. The maximum error in the positioning with this procedure was estimated to be $\pm 1 \mathrm{~mm}$ in each direction. For total scatter output factors, several measurements were taken, repeating the positioning process for the spherical diode, and no response difference was observed (within experimental error), so we consider that the uncertainty in the positioning of the diode introduced no significant error. 


\section{RESULTS AND DISCUSSION}

Using a calibrated mammogram, we measured the diameter of six Spheral ${ }^{\mathbb{R}}$ diodes, resulting in an average of 1.85 $\pm 0.02 \mathrm{~mm}$ (one standard deviation), according to manufacturer's specifications. ${ }^{38,39}$ This size enables adequate spatial resolution.

\section{A. Sensitivity variation with accumulated dose}

In Fig. 3 we represent the change in response of the Sphelar ${ }^{\circledR}$ diode after consecutive irradiations. As it has been explained before, we used three different diodes. After a preirradiation of $4 \mathrm{kGy}$, we obtained a constant decay rate of about $5 \% / \mathrm{kGy}$ for $15 \mathrm{MV}$ photon beam; after $12 \mathrm{kGy}$, the decay rate is less than $1.5 \% / \mathrm{kGy}$ at $15 \mathrm{MV}$, and less than $0.7 \% / \mathrm{kGy}$ at $6 \mathrm{MV}$. This value is similar to those of some commercial in vivo detector as IBA 3G-pSi (4.8\% at $15 \mathrm{MV})$, Sun Nuclear IsoRad and QED (0.5\% at $6 \mathrm{MV})$, Nuclear Associates VeriDose $(1.5 \%)$. In any case, the estimation of response decrease
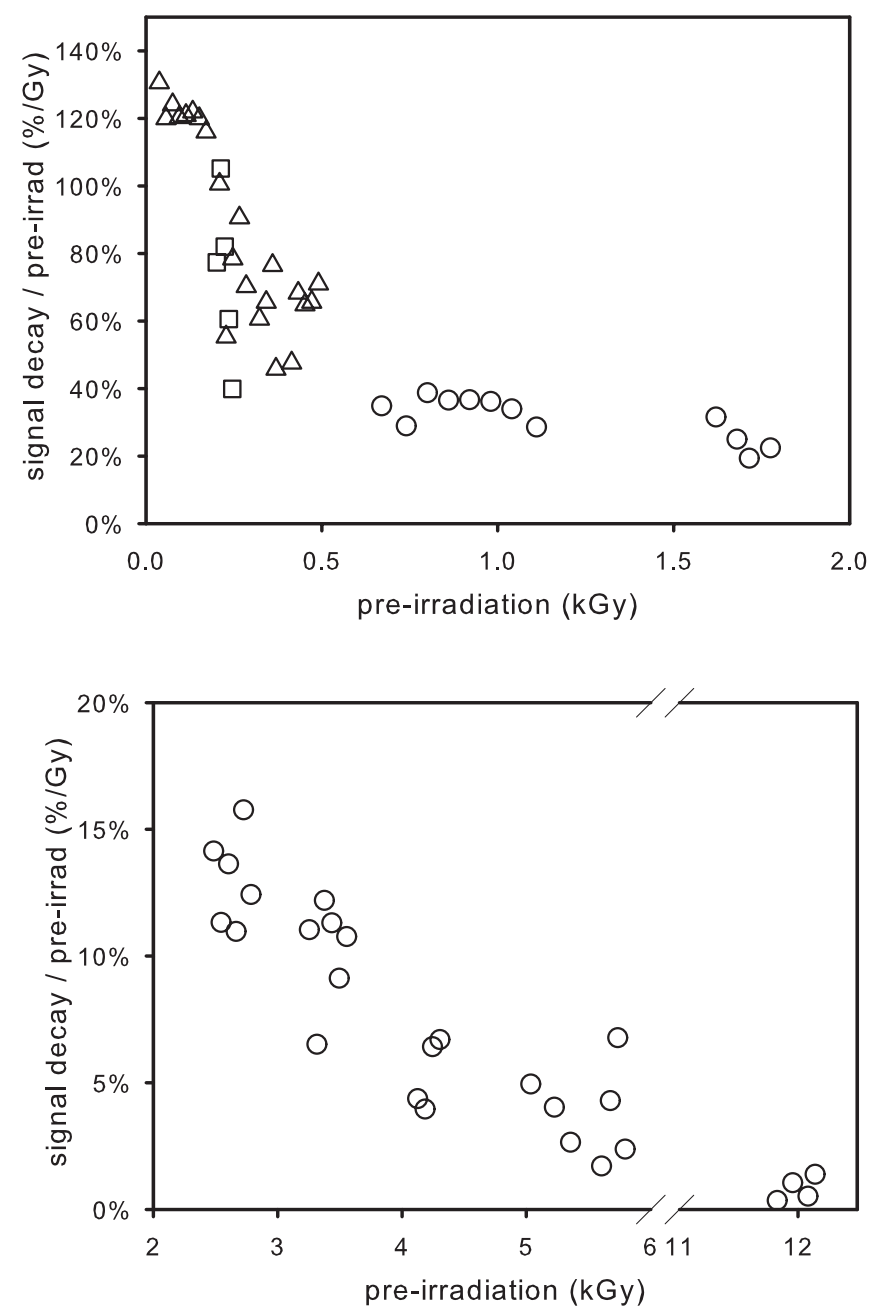

FIG. 3. Decrease rate of the signal from the Sphelar ${ }^{\circledR}$ diode with respect to the previously irradiated dose, from 0 to $2 \mathrm{kGy}$ (up) and from 2 to $12.5 \mathrm{kGy}$ (down), for $15 \mathrm{MV}$ photon beam. Different set of points correspond to different diodes. in point dose measurements in the range of $150-1000 \mathrm{cGy}$, which is usual in radiation therapy, was less than $0.01 \%$.

With respect to sensitivity, after $12 \mathrm{kGy}$ preirradiation the Sphelar ${ }^{\circledR}$ had a response above $150 \mathrm{nC} /$ Gy for $15 \mathrm{MV}$ photons and $160 \mathrm{nC} / \mathrm{Gy}$ for $6 \mathrm{MV}$ photons. This value is higher than those of some commercial in vivo detector, of similar size or bigger, as IBA 3G-pSi ( $2 \mathrm{~mm}$ diameter, $25 \mathrm{nC} / \mathrm{Gy}$ ), Sun Nuclear IsoRad (1.4 mm diameter, $27 \mathrm{nC} / \mathrm{Gy})$, Sun Nuclear QED $(0.8 \times 0.8 \mathrm{~mm}, 32 \mathrm{nC} / \mathrm{Gy})$, and Nuclear Associates VeriDose ( $8 \mathrm{~mm}$ diameter, $150 \mathrm{nC} / \mathrm{Gy}$ ).

\section{B. Angular response}

The usual way to check the angular response of a diode is to measure the signal around one of its axes, the most favorable. As the diode Sphelar ${ }^{\circledR}$ is spherically symmetric, angular response should be studied in all possible positions. We performed measurements by rotating the $\mathrm{x}$ ray beam around the three axes of the diode, angles $\alpha, \beta$, and $\gamma$, as described in Fig. 2. Results are shown in Fig. 4. For each one of the three positions of the diode, five series of measurements were taken, each one of 18 beam angles. Each series of
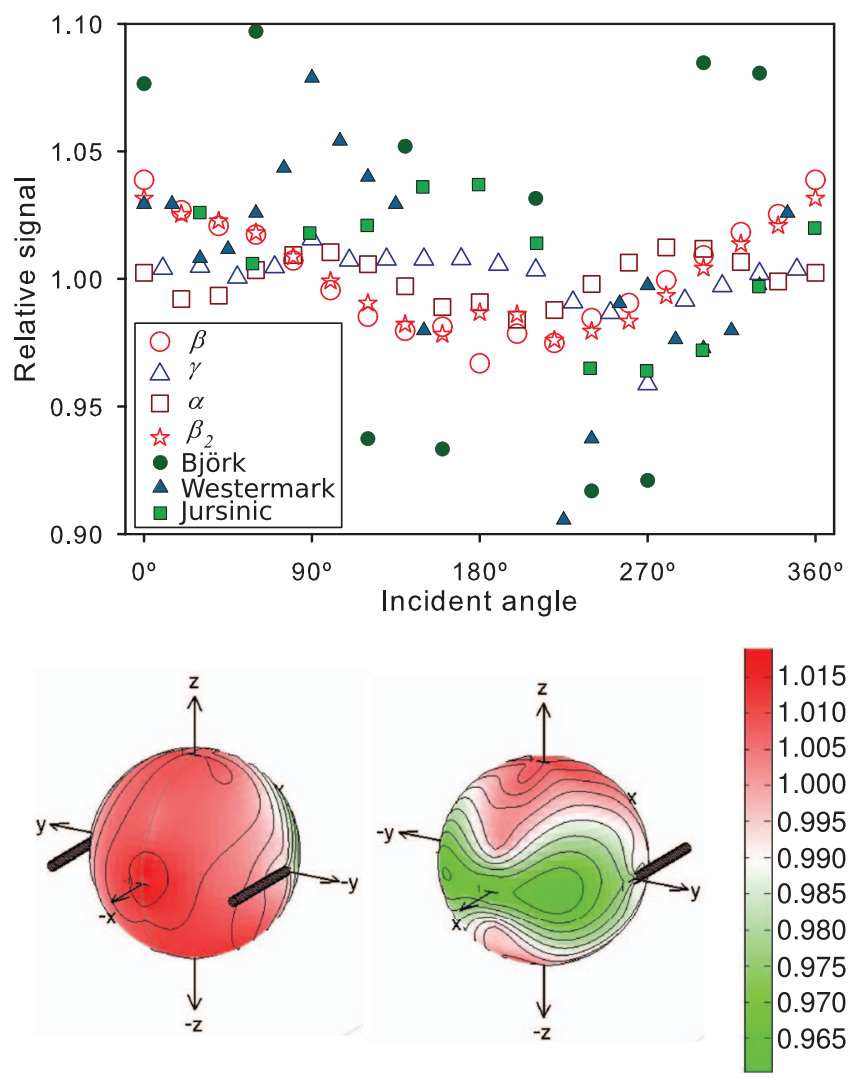

FIG. 4. Top: Radiation sensitivity of Sphelar ${ }^{\circledR}$ diode as a function of the angle of incidence of x-ray radiation. For each angle signals are normalized to their mean value. Dots are mean value of five measurements; standard deviations of these values are always $<0.002$, and are not represented. Data of other three authors are shown for comparison. Bottom: 3D reconstruction of the radiation sensitivity over the diode's surface. The relative sensitivity is represented as dark grey for values larger than 1, and light grey for values smaller than 1 . Two symmetrical views are presented. The connecting wires are represented parallel to the $\mathrm{X}$-axis. 
measurements was normalized to the average of 18 measurements, in order to reduce the effect of signal decrease with accumulated dose (always less than $0.2 \%$ ). Differences between maximum and minimum values are $3 \%$ turning around $X$-axis $(\alpha), 6 \%$ around $Y$-axis $(\gamma)$, and $7 \%$ around $Z$-axis $(\beta)$. Extreme values turning around $Y$ and $Z$-axis were those in which direct radiation went through the cable $(9 \mathrm{~cm}$ long, approximately). To test the cable effect, other set of measurements around $\beta$ angle was done with other diode, performing a setup with the cable in the axis of the phantom, so that the beam did not cross the cable. This setup involved the position of the diode in the phantom to be less accurate: these measurements were done only to test the cable effect. We plotted them in Fig. 4 as $\beta_{2}$. It can be seen that former extreme values are not present, so we confirm that they were due to the effect of the cable. If extreme values are not taken into account, differences between maximum and minimum values decrease to $2 \%$ around $Y$-axis and $5 \%$ around $Z$-axis. When turning around $X$-axis, direct beam does not cross the cable, and the main effect probably corresponds to the small area without an $\mathrm{n}^{+}$diffusion layer around the anode (signal decrement in the angles close to $0^{\circ}$ and $180^{\circ}$ ). That, and the presence of the electrodes, is the probable cause of the difference in the angular behavior with $\beta$ and $\gamma$. Each point in Fig. 4 is the average of 5 measurements. Error bars are not drawn, because the standard deviation is always less than 0.002 . These results are compared with those of other authors, in similar geometry: Björk et al. ${ }^{42}$ mounted on central axis of cylindrical polyethylene phantom, $6 \mathrm{MeV}$ electrons; Westmark et al. ${ }^{43}$ mounted on central axis of cylindrical water phantom, $18 \mathrm{MV}$ x rays; Jursinic ${ }^{26}$ mounted on central axis of cylindrical M3 phantom, $6 \mathrm{MV} \mathrm{x}$ rays.

\section{C. Instantaneous dose rate (or dose per pulse) dependence}

The Oncor Impression Plus used has a pulse width (at $71 \%$ of maximum) of $3.1 \mu \mathrm{s}$ and a pulse repetition period of $4.4 \mathrm{~ms}$. It takes $12 \mathrm{~s}$ to deliver $100 \mathrm{MU}$ (500 MU/min). A Farmer chamber placed at the side of the diode was used to measure the dose absorbed with $100 \mathrm{MU}$, at different SSDs, to obtain several values of dose per pulse. Figure 5 shows the diode response as a function of the dose per pulse: absorbed dose divided by the number of pulses in $100 \mathrm{MU}$. Values are normalized to 1.0 at the SSD of $100 \mathrm{~cm}$.

\section{D. Measurements in large fields}

We performed measurements of total scatter factors $S_{c p}$ for photon beams of nominal energies of 6 and $15 \mathrm{MV}$, in square fields with field size between $3 \times 3$ and $20 \times 20 \mathrm{~cm}$ in the RW3 phantom, using the $0.125 \mathrm{~cm}^{3}$ ionization chamber. Two measurements were taken for each nominal energy, and the average was calculated for each beam aperture. For each field, two measurements were performed with the EFD diode in the water tank and eight with the Sphelar ${ }^{\circledR}$ diode in the water equivalent plastic phantom. In Fig. 6 we represent the total

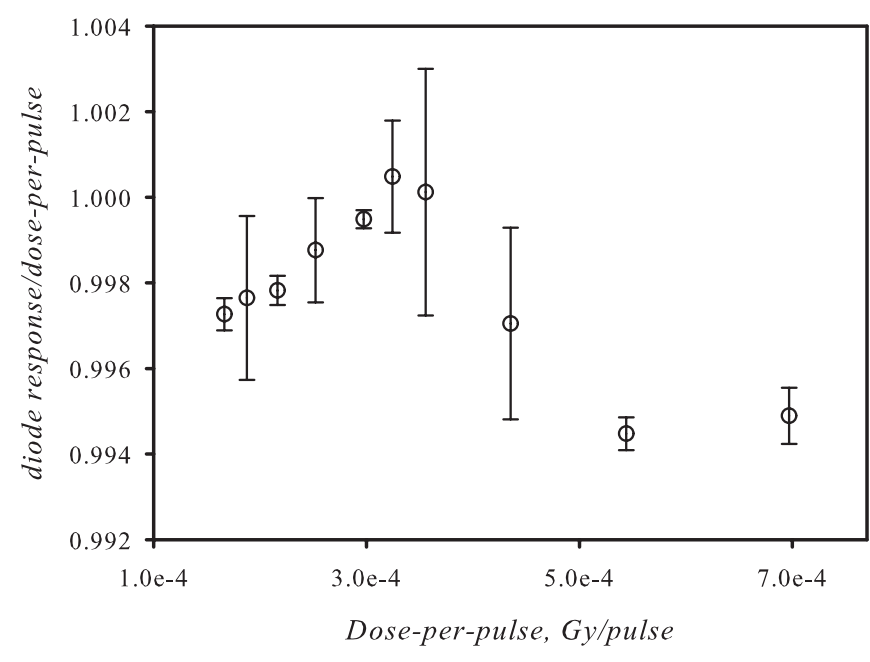

FIG. 5. Dose response of Sphelar ${ }^{\circledR}$ diode as a function of dose per pulse of a $15 \mathrm{MV}$ x-ray beam. Values normalized to 1.0 at $3.56 \times 10^{-4} \mathrm{~Gy} /$ pulse. The error bars show the standard deviation.
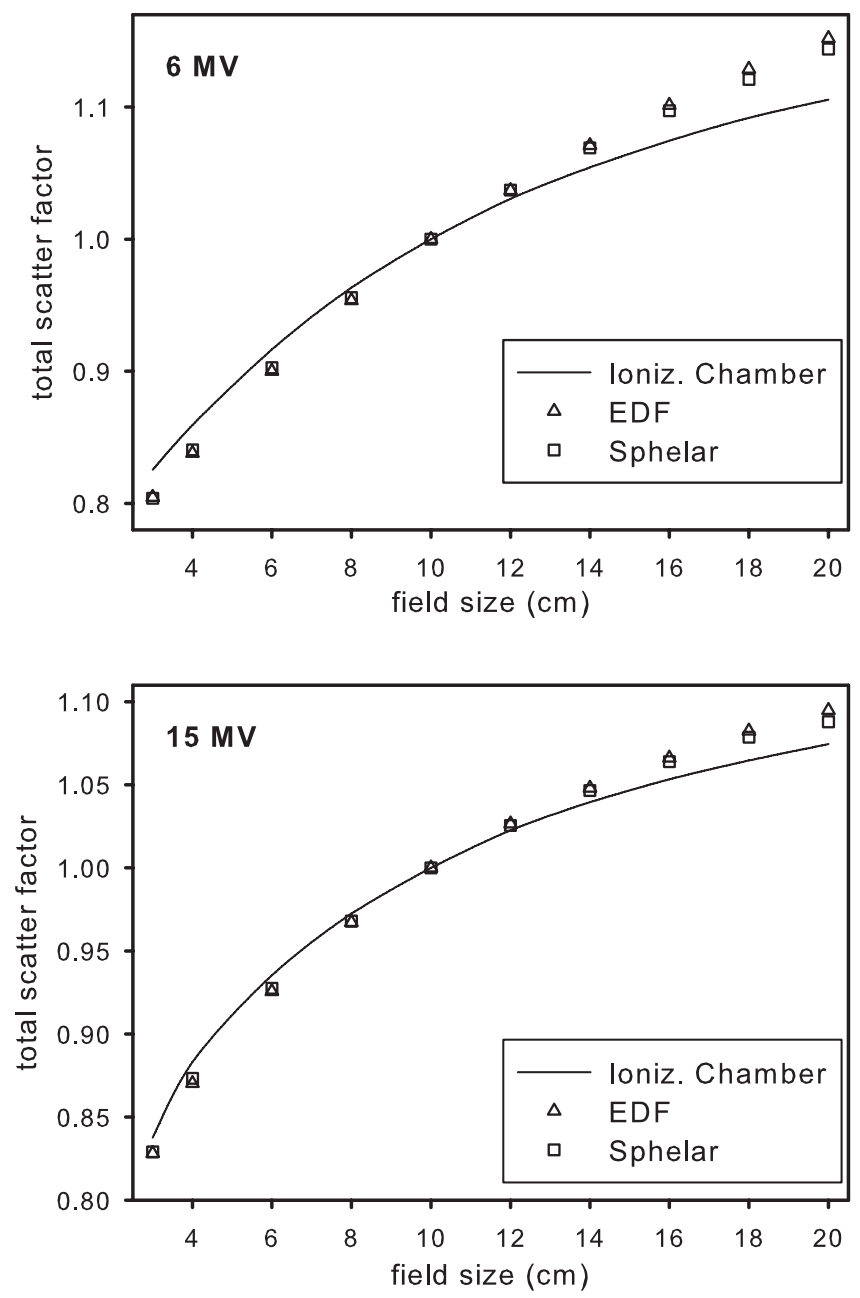

FIG. 6. Measurement of $S_{c p}$ for 6 and $15 \mathrm{MV}$ nominal energy photon beams, using a $0.125 \mathrm{cc}$ ionization chamber PTW 31002 (mean of two measurements), EFD diode (mean of two measurements), Sphelar ${ }^{\circledR}$ diode (mean of eight measurements). Error bars are not drawn because standard deviations of Sphelar values are less than 0.01 . 
scatter factor $S_{c p}$ calculation, normalized to the $10 \times 10 \mathrm{~cm}$ field value. It can be observed that the Sphelar ${ }^{\circledR}$ diode has a response similar to that of the EFD, which was specifically designed for that range of energies. Both of them show an overresponse in larger fields. The number of Compton-scattered low-energy photons increases with the field size and they interact by photoelectric effect in silicon due to its higher cross section for this effect, which increases with the mean atomic number $\mathrm{Z}$. This effect is described in the bibliography. ${ }^{1,44,45}$ On the other hand, small fields have less scattered photons. We will dedicate a specific section of this paper to show that it is possible to correct this effect in the Sphelar ${ }^{\circledR}$ diode using a metallic sleeve, as it is usually done in diodes designed to make measurements in photon beams. ${ }^{46}$

As far as repeatability is of concern, standard deviations of the measurements for each field with Sphelar ${ }^{\circledR}$ diode were always below 0.01 .

\section{E. Measurements in small fields}

We performed measurements of total scatter factors $S_{c p}$ for field sizes between $1 \times 1$ and $6 \times 6 \mathrm{~cm}$, in water equivalent solid phantom, employing the PTW 31016 PinPoint chamber. In the water tank we performed the same measurements with the PinPoint chamber, Scanditronix EFD (unshielded), SFD (stereotactic, also unshielded), and the Sphelar ${ }^{\circledR}$ One diodes. The reference field size for those measurements was $4 \times 4 \mathrm{~cm}$. The SFD measurements, averaged over three readings, are the reference values to which the other measurements are compared in Fig. 7. As we can see, the spherical diode had a similar response to the other three detectors that had been specially designed for this type of measurement, except in smaller fields, in which an under-response can be observed. The PinPoint chamber is less reliable for field sizes smaller than $2 \mathrm{~cm} \cdot{ }^{47,48}$ As before, standard deviations of the five measurements for each field with the Sphelar ${ }^{\circledR}$ diode were always less than 0.01 , which shows good repeatability.

Differences among measurements of $S_{c p}$ with Sphelar ${ }^{\circledR}$ in plastic phantom (Sec. 3.D) and in water (this section), were always less than $0.5 \%$, for fields between $6 \times 6 \mathrm{~cm}$ and $3 \times 3 \mathrm{~cm}$. This fact suggests that there was charged particle equilibrium in the diode inside the solid phantom, as was supposed, in spite of the fact that the slab insert was not designed specifically for the diode, so there was a thin layer of air (less than $1 \mathrm{~mm}$ ) between the diode and the holder, which in any case is much shorter than the secondary electron range.

\section{F. Diode compensation/shielding}

As we already mentioned, silicon diodes exhibit an overresponse to low energy Compton scattered photons in large fields. Theoretical models that take into account this effect have been developed, ${ }^{44,46}$ but it is common practice among the manufacturers to add a layer of a high atomic number material covering all the silicon crystal except the doped surface to compensate for this effect. ${ }^{49-51}$ This compensation is purely
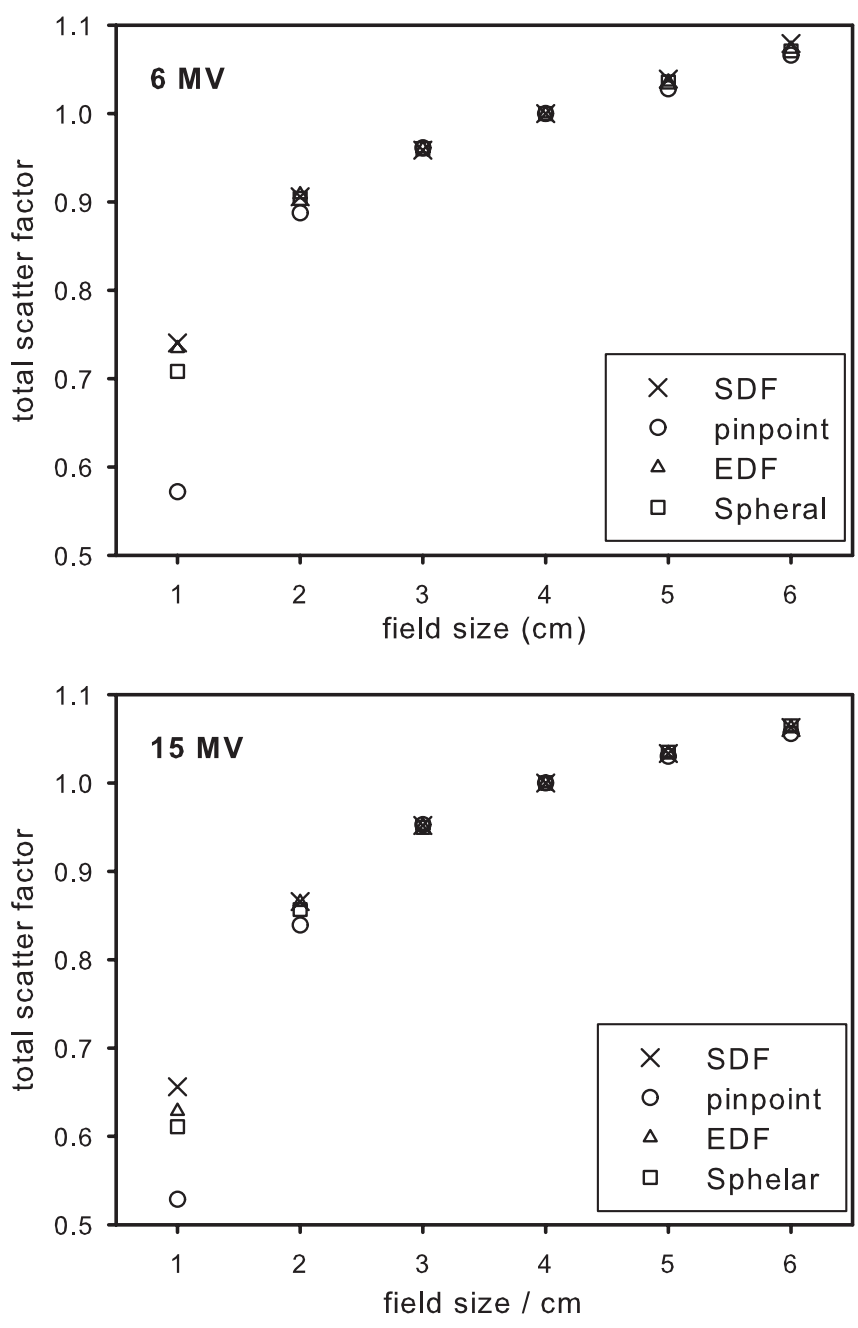

FIG. 7. Measurement of $S_{c p}$ for 6 and $15 \mathrm{MV}$ nominal energy photon beams, using a SFD diode (mean of three measurements), EFD diode (mean of three measurements), Sphelar ${ }^{\circledR}$ diode (mean of five measurements), and PTW 31016 PinPoint chamber (mean of two values). Error bars are not represented because standard deviations of Sphelar ${ }^{\circledR}$ values are less than 0.01 .

empirical. In order to demonstrate that the Sphelar ${ }^{\circledR}$ diode can be compensated to measure dosimetric $\mathrm{x}$-ray properties in large fields, we measured total scatter factors for field sizes between $3 \times 3$ and $20 \times 20 \mathrm{~cm}$, covering the diode successively with several layers of different thicknesses of lead ( 0.4 and $0.9 \mathrm{~mm})$ and brass $(0.1,0.3$, and $0.5 \mathrm{~mm})$. In our case, the diode collects signal from all directions, so it is necessary to cover it completely with the shielding. In Fig. 8 we can observe that a $0.5 \mathrm{~mm}$ brass layer achieves the compensation required to match the diode measurements with those of the ionization chamber employed within an error of $1 \%$ for the $6 \mathrm{MV}$ photons, and with a negligible difference for $15 \mathrm{MV}$ photons (except for small fields). A $0.4 \mathrm{~mm}$ layer of lead results in too much shielding. We show some example values in Table I. From these results we can conclude that it is possible to find an adequate empirical correction for the Sphelar ${ }^{\circledR}$ diode that allows large fields to be measured with enough accuracy in the therapeutic range of megavoltage $\mathrm{x}$ rays. 

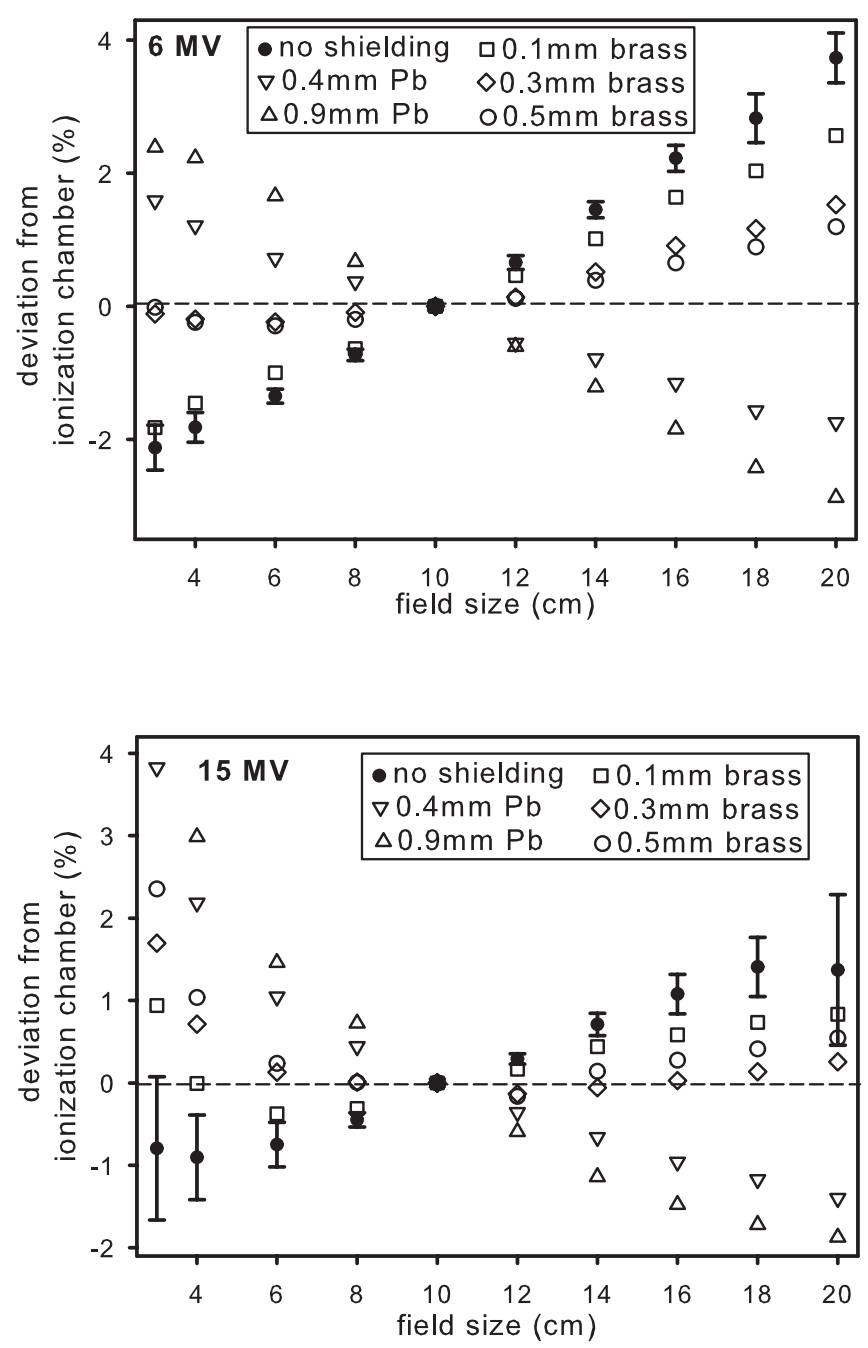

FIG. 8. Comparison among $S_{c p}$ values, relative to those measured with the ionization chamber $\left(\left[S_{c p}-S_{c p, i o n}\right] / S_{c p, i o n}(10)\right)$, for both 6 and $15 \mathrm{MV}$ photon beams as a function of the beam aperture, measured in water equivalent solid phantom with the Sphelar diode (average of 10 measurements, bar error corresponding to one standard deviation), and with different amount of metal layer for shielding.

\section{G. Signal reproducibility}

To test the signal reproducibility, we performed 44 sets of five measurements of an irradiation with calculated dose of $1.25 \mathrm{~Gy}$ and with energy of $15 \mathrm{MV}$. The maximum typical deviation (standard deviation/mean) obtained was $0.3 \%$. The median of typical deviation was $0.08 \%$.

\section{CONCLUSIONS}

A preliminary study was performed, on the feasibility of using a new diode with spherical symmetry for the measurement of relative dose in MV beams. To do this, we used a set of low cost photodiodes, without adding any modification other than a resin coating to make one of them waterproof. We conclude that, even without modifying the diodes, they have a sufficiently good response to be employed in clinical and research applications.

The most outstanding result is that, as was expected owing to its geometry, the angular dependence of the spherical diodes is slightly lower than that of other detectors specifically designed to achieve good directional uniformity, ${ }^{21}$ as it can be seen in Fig. 4. Besides, that dependence is low in all directions of the space, not only in some favorable directions. Moreover, as it is capable of detecting incident radiation from all directions, sensitivity ( $150 \mathrm{nC} / \mathrm{Gy}$ for photons of nominal energy of $15 \mathrm{MV}$ and 160 for $6 \mathrm{MV}$, after $12 \mathrm{kGy}$ ) is better than that of other commercial in vivo diodes of similar size. So, smaller diodes with the same spherical geometry would be sensitive enough to produce a detectable charge, and would allow better spatial resolution.

The response variation with accumulated dose was found to be similar to that of other MV-dedicated commercial or experimental diodes, and it is small enough to be considered negligible in conventional relative dose measurements of a few Gy. Variation with dose per pulse was less than $\pm 0.4 \%$. Its reproducibility and measurement accuracy are similar to those of other detectors commonly employed, both in large fields and in small fields down to $2 \times 2 \mathrm{~cm}$. The response worsens for fields smaller than $2 \times 2 \mathrm{~cm}$. Its over-response in larger fields could be corrected empirically by using shielding. The feasibility of use these detectors for small beam dosimetry still remains unanswered, requiring a deeper study by Monte Carlo to model the variation of its response with field size.

The authors specially stress the good angular response in the MV range. This is a promising result for its application to patient in vivo dose measurements and in arc measurements.

\section{ACKNOWLEDGMENTS}

The authors acknowledge financial support of Spanish Government through Contract No. FIS2011-24642 and Fundación Mutua Madrileña. The authors also acknowledge Mevion Technology S.L. (Soria, Spain) for their assistance with the irradiation of the diodes at high doses.

TABLE I. Some $S_{c p}$ example values to test shielding effect.

\begin{tabular}{lcccccc}
\hline \hline Field size & 6 MV Ion Chamber & 6 MV no shield & 6 MV 0.5 mm brass & 15 MV Ion Chamber & 15 MV no shield & 15 MV 0.5 mm brass \\
\hline $3 \times 3$ & 0.825 & 0.804 & 0.825 & 0.838 & 0.830 & 0.861 \\
$6 \times 6$ & 0.917 & 0.903 & 0.914 & 0.935 & 1.928 & 0.938 \\
$14 \times 14$ & 1.054 & 1.069 & 1.058 & 1.053 & 1.064 & 1.056 \\
$20 \times 20$ & 1.106 & 1.143 & 1.118 & 1.074 & 1.080 \\
\hline \hline
\end{tabular}


a) Author to whom correspondence should be addressed. Electronic mail: bbarbes@unav.es

${ }^{1}$ I. J. Das, G. X. Ding, and A. Ahnesjö, "Small fields: Nonequilibrium radiation dosimetry," Med. Phys. 35, 206-215 (2008).

${ }^{2}$ International Commission of Radiation Units and Measurements, Prescribing, Recording, and Reporting Photon-Beam Intensity-Modulated Radiation Therapy (IMRT), Journal of the ICRU Vol. 10 (Oxford University Press, 2010), p. 1.

${ }^{3}$ P. Andreo, D. T. Burns, K. Hohlfeld, M. S. Huq, T. Kanai, F. Laitano, V. G. Smyth, and S. Vynckier, "Absorbed dose determination in external beam radiotherapy," IAEA Technical Report Series No. 398 (International Atomic Energy Agency, Vienna, 2000).

${ }^{4}$ T. Guldbrandsen and C. B. Madsen, "Radiation dosimetry by means of semiconductors," Acta Radiol. 58(3), 226-232 (1961).

${ }^{5} \mathrm{~K}$. Scharf and J. H. Sparrow, "Steady-state response of silicon radiation detectors of diffused p-n junction-type to x-rays. 1R: Photo-voltage mode of operation," J. Res. Natl. Bur. Stand. 68A, 683-701 (1964).

${ }^{6}$ R. P. Parker, "Semiconductor nuclear radiation detectors," Phys. Med. Biol. 15, 605-620 (1970)

${ }^{7}$ S. C. Klevenhagen, "Temperature response of silicon surface barrier semiconductor detector operated in the DC-short circuit configuration," Acta Radiol. 12, 124-144 (1973).

${ }^{8} \mathrm{~J}$. Van Dam and G. Marinello, Methods for in vivo Dosimetry in External Radiotherapy, ESTRO Booklet on Physics for Clinical Radiotherapy No. 1. Garant (Leuven-Apeldoorn, 1994), see also PDF version at http://www.estroweb.org.

${ }^{9}$ G. Rikner and E. Grusell, "Effect of radiation damage on p-type silicon detectors," Phys. Med. Biol. 28, 1261-1267 (1983).

${ }^{10} \mathrm{G}$. Rikner and E. Grusell, "General specification for silicon semiconductors for use in radiation dosimetry," Phys. Med. Biol. 32, 1109-1117 (1987).

${ }^{11}$ E. York et al., "Diode in vivo dosimetry for patients receiving external beam radiation therapy," AAPM Report No. 87 (American Association of Physicists in Medicine, College Park, MD, 2005).

${ }^{12} \mathrm{M}$. Essers and B. J. Mijheer, "In vivo dosimetry during external photon beam radiotherapy," Int. J. Radiat. Oncol., Biol., Phys. 43, 245-259 (1999).

${ }^{13}$ D. Huyskens et al., European Society for Therapeutic Radiology and Oncology, Booklet No. 5, 2001.

${ }^{14}$ F. H. Attix, Introduction to Radiological Physics and Radiation Dosimetry (Wiley, New York, 1986), pp. 395-437.

${ }^{15}$ E. Pappas, L. Petrokokkinos, A. Angelopoulos, T. G. Maris, M. Kozicki, I. Dalezios, and V. Kouloulias, "Relative output factor measurements of a $5 \mathrm{~mm}$ diameter radiosurgical photon beam using polymer gel dosimetry," Med. Phys. 32(6), 1513-1520 (2005).

${ }^{16}$ F. Chen, C. F. O. Graeff, and O. Baffa, "K-band EPR dosimetry: Smallfield beam profile determination with miniature alanine dosimeter," Appl. Radiat. Isot. 62, 267-271 (2005).

${ }^{17}$ K. A. Paskalev, J. P. Seuntjens, H. J. Patrocinio, and E. B. Podgorsak, "Physical aspects of dynamic stereotactic radiosurgery with very small photon beams (1.5 and $3 \mathrm{~mm}$ in diameter)," Med. Phys. 30(2), 111-118 (2003).

${ }^{18}$ C. De Angelis, S. Onori, M. Pacilio, G. A. Cirrone, G. Cuttone, L. Raffaele, M. Bucciolini, and S. Mazzocchi, "An investigation of the operating characteristics of two PTW diamond detectors in photon and electron beams," Med. Phys. 29, 248-254 (2002).

${ }^{19}$ Y. W. Vahc, W. K. Chung, K. R. Park, J. Y. Lee, Y. H. Lee, O. Kwon, and S. Kim, "The properties of the ultramicrocylindrical ionization chamber for small field used in stereotactic radiosurgery," Med. Phys. 28, 303-309 (2001).

${ }^{20}$ M. Soubra, J. Cygler, and G. Mackay, "Evaluation of a dual bias dual metal oxide-silicon semiconductor field effect transistor detector as radiation detector," Med. Phys. 21, 567-572 (1994).

${ }^{21} \mathrm{P}$. A. Jursinic, "Characterization of optically stimulated luminescent dosimeters, OSLDs, for clinical dosimetric measurements," Med. Phys. 34, 4594-4604 (2007).

${ }^{22}$ G. F. Knoll, Radiation Detection and Measurement, 4th ed. (John Wiley and Sons Inc, 2010), Chap. 11.

${ }^{23}$ R. F. Pierret, Advanced Semiconductor Fundamentals, 2nd ed. (PrenticeHall, New York, 2002).

${ }^{24}$ J. Shi, W. E. Simon, and T. C. Zhu, "Modeling the instantaneous dose rate dependence of radiation diode detectors," Med. Phys. 30, 2509-2519 (2003).
${ }^{25}$ J. Shi, W. E. Simon, L. Ding, and D. Saini, "Important issues regarding diode performance in radiation therapy applications," Digest of Papers of the 2000 World Congress on Medical Physics and Biomedical Engineering and the Proceedings of the 22nd Annual International Conference of the IEEE Engineering in Medicine and Biology Society, 0-7803-6468-6 (C) 2000 IEEE, Chicago (2000).

${ }^{26} \mathrm{P}$. A. Jursinic, "Angular dependence of dose sensitivity of surface diodes," Med. Phys. 36(6), 2165-2171 (2009).

${ }^{27}$ J. Shi, W. E. Simon, L. Ding, D. Saini, and S. Rose, "Effects of buildup thickness and material to diode detector SSD dependence.” Med. Phys. 26, 1127 (1999).

${ }^{28}$ C. B. Saw, J. Shi, and D. H. Hussey, "Energy dependence of a new solid state diode for low energy photon beam dosimetry," Med. Dosim. 23, 95 97 (1998).

${ }^{29}$ D. Georg, B. De Ost, M. T. Hoornaert, P. Pilette, J. Van Dam, M. Van Dycke, and D. Huyskens, "Buildup modification of commercial diodes for entrance dose measurements in "higher energy" photon beams," Radiother. Oncol. 51, 249-256 (1999).

${ }^{30}$ J. R. Greig, R. W. Miller, and P. Okunieff, "An approach to dose measurement for total body irradiation,” Int. J. Radiat. Oncol., Biol., Phys. 36 463-468 (1996).

${ }^{31}$ R. Alecu, M. Alecu, and T. G. Ochran, "A method to improve the effectiveness of diode in vivo dosimetry," Med. Phys. 25, 746-749 (1998).

${ }^{32}$ J. N. Eveling, A. M. Morgan, and W. G. Pitchford, "Commissioning a ptype silicon diode for use in clinical electron beams," Med. Phys. 26, 100107 (1999).

${ }^{33}$ J. G. Wierzbicki and D. S. Waid, "Large discrepancies between calculated $\mathrm{D}_{\max }$ and diode readings for small field sizes and small SSDs of $15 \mathrm{MV}$ photon beams," Med. Phys. 25, 245-246 (1998).

${ }^{34}$ A. Sen, E. I. Parsai, S. W. McNeeley, and K. M. Ayyangar, "Quantitative assessment of beam perturbations caused by silicon diodes used for in vivo dosimetry," Int. J. Radiat. Oncol., Biol., Phys. 36, 205-211 (1996).

${ }^{35}$ A. Broisman and G. Shani, "Application of spherical micro diodes for brachytherapy dosimetry," Radiat. Meas. 46, 334-339 (2011).

${ }^{36}$ A. S. Saini and T. C. Zhu, "Temperature dependence of commercially available diode detectors," Med. Phys. 29, 622-630 (2002).

${ }^{37}$ R. Alfonso, P. Andreo, R. Capote, M. Saiful Huq, W. Kilby, P. Kjäll, T. R. Mackie, H. Palmans, K. Rosser, J. Seuntjens, W. Ullrich, and S. Vatnitsky, "A new formalism for reference dosimetry of small and nonstandard fields," Med. Phys. 35(11), 5179-5186 (2008).

${ }^{38}$ J. Nakata, N. Kuratani, H. Tomozawa, Y. Nishimura, N. Yokogawa, I. Inagawa, and K. Nishida, "Ge and GaSb seedless crystal growth under microgravity conditions," Space Forum 6, 213-220 (2000).

${ }^{39}$ J. Nakata, Spherical Solar Cells Solve Issue of 3-D Sunlight Reception, Asia Electronics Industry (AEI), Dempa Publications (2003), pp. 45-46.

${ }^{40}$ N. Kogo, K. Taira, H. Kikuchi, N. Kumagai, N. Kuratani, I. Inagawa, S. Imoto, and J. Nakata, "Three dimensional light capture of spherical cells," in 15th International Photovoltaic Science and Engineering Conference (PVSEC-15), Shanghai Scientific \& Technical Publishers, Shanghai China (2005), pp. 202-203.

${ }^{41}$ R. Nath, L. L. Anderson, G. Luxton, K. A. Weaver, J. F. Williamson, and A. S. Meigooni, "Dosimetry of interstitial brachytherapy sources: Recommendations of the AAPM Radiation Therapy Committee Task Group No. 43," Med. Phys. 22, 209-234 (1995).

${ }^{42}$ P. Björk, T. Knöös, and P. Nilsson, "Comparative dosimetry of diode and diamond detectors in electron beams for intraoperative radiation therapy," Med. Phys. 27, 2580-2588 (2000).

${ }^{43}$ M. Westermark, J. Arndt, B. Nilsson, and A. Brahme, "Comparative dosimetry in narrow high-energy photon beams," Phys. Med. Biol. 45, 685-702 (2000).

${ }^{44} \mathrm{Z}$. Yin, R. P. Hugtenburg, and A. H. Beddoe, "Response corrections for solid-state detectors in megavoltage photon dosimetry," Phys. Med. Biol. 49, 3691-3702 (2004).

${ }^{45}$ I. Griessbach, M. Lapp, J. Bohsung, and G. Gademann, "Dosimetric characteristics of a new unshielded silicon diode and its application in clinical photon and electron beams," Med. Phys. 32(12), 3750-3754 (2005).

${ }^{46}$ O. A. Sauer and J. Wilbert, "Measurement of output factors for small photon beams," Med. Phys. 34, 1983-1988 (2007).

${ }^{47}$ C. Martens, C. De Wagter, and W. De Neve, "The value of the PinPoint ion chamber for characterization of small field segments used in intensitymodulated radiotherapy," Phys. Med. Biol. 45, 2519-2530 (2000). 
${ }^{48}$ S. Agostinelli, S. Garelli, M. Piergentili, and F. Foppiano, "Response to high-energy photons of PTW31014 PinPoint ion chamber with a central aluminum electrode," Med. Phys. 35, 3293-3301 (2008).

${ }^{49} \mathrm{G}$. Rikner and E. Grusell, "Selective shielding of a p-Si detector for quality independence," Acta Radiol. Oncol. 24, 65-69 (1985).
${ }^{50}$ L. D. Gager, A. E. Wright, and P. R. Almond, "Silicon diode detectors used in radiological physics measurements. Part I: Development of an energy compensating shield," Med. Phys. 4(6), 494-498 (1977)

${ }^{51}$ A. E. Wright and L. D. Gager, "Silicon diode detectors used in radiological physics measurements. Part II: Measurement of dosimetry data for highenergy photons," Med. Phys. 4(6), 499-502 (1977). 